\title{
Human navigation network: the intrinsic functional organization and behavioral relevance
}

\author{
Xiang-Zhen Kong ${ }^{1} \cdot \mathrm{Xu} \mathrm{Wang}^{1} \cdot \mathrm{Yi} \mathrm{Pu}^{3,4} \cdot$ Lijie Huang $^{1} \cdot \mathrm{Xin} \mathrm{Hao}^{1} \cdot$ \\ Zonglei Zhen ${ }^{1} \cdot$ Jia Liu $^{2}$
}

Received: 2 January 2016/ Accepted: 26 May 2016/Published online: 3 June 2016

(C) Springer-Verlag Berlin Heidelberg 2016

\begin{abstract}
Spatial navigation is a crucial ability for living. Previous work has revealed multiple distributed brain regions associated with human navigation. However, little is known about how these regions work together as a network (referred to as navigation network) to support flexible navigation. In a novel protocol, we combined neuroimaging meta-analysis, and functional connectivity and behavioral data from the same subjects. Briefly, we first constructed the navigation network for each participant, by combining a large-scale neuroimaging metaanalysis (with the Neurosynth) and resting-state functional magnetic resonance imaging. Then, we investigated multiple topological properties of the navigation networks, including small-worldness, modularity, and highly connected hubs. Finally, we explored the behavioral relevance of these intrinsic properties in a large sample of healthy young adults $(N=190)$. We found that navigation networks showed small-world and modular organization at global level. More importantly, we found that increased
\end{abstract}

Xiang-Zhen Kong

xiangzhen.kong@outlook.com

$\triangle$ Jia Liu

liujia@bnu.edu.cn

1 State Key Laboratory of Cognitive Neuroscience and Learning \& IDG/McGovern Institute for Brain Research, Beijing Normal University, 19 Xinjiekouwai St, Haidian District, Beijing 100875, China

2 School of Psychology, Beijing Normal University, 19 Xinjiekouwai St, Haidian District, Beijing 100875, China

3 ARC Centre of Excellence in Cognition and Its Disorders, Macquarie University, Sydney, NSW 2109, Australia

4 Department of Cognitive Science, Macquarie University, Sydney, NSW 2109, Australia small-worldness and modularity of the navigation network were associated with better navigation ability. Finally, we found that the right retrosplenial complex (RSC) acted as one of the hubs in the navigation network, and that higher betweenness of this region correlated with better navigation ability, suggesting a critical role of the RSC in modulating the navigation network in human brain. Our study takes one of the first steps toward understanding the underlying organization of the navigation network. Moreover, these findings suggest the potential applications of the novel approach to investigating functionally meaningful networks in human brain and their relations to the behavioral impairments in the aging and psychiatric patients.

Keywords Spatial navigation - Functional connectivity · Individual differences · Connectomics

\section{Introduction}

Spatial navigation is a crucial ability for living, since wayfinding and environment exploration always happen in our daily life. Previous neuroimaging studies have identified multiple regions distributed across the brain, which have been implicated to be associated with different functions for navigation (see Wolbers and Hegarty 2010 for a review). For example, the hippocampus has been proposed to support a cognitive map (O'Keefe and Dostrovsky 1971), while the parahippocampal place area (PPA) (Epstein et al. 1999) and the retrosplenial complex (RSC) (Maguire 2001) have been shown responsible for visual scene processing. In addition to scene processing, the RSC has recently been implicated in anchoring our sense of direction to local environment (Marchette et al. 2014). Besides, the parietal and frontal regions have been shown 
to be necessary to generate spatial imagery and make orientation decisions (Harvey et al. 2012; Mellet et al. 1995), and maintain actively the goal destination in working memory for use in navigation (Ciaramelli 2008), respectively. Supplemental to the interest on the common functionality shared by individuals, another fundamental question is how navigation-related neural substrates account for individual differences in the ability to navigate the world.

Following the traditional functional localization, studies on individual differences in navigation have mainly focused on local variability in anatomical and functional features of these regions. For example, regional gray matter volumes in the hippocampus, parahippocampal cortex and prefrontal regions have been showed associated with navigation performance (e.g., Wegman et al. 2014; Brown et al. 2014; Moffat et al. 2007). Similarly, functional activity in the hippocampus and parahippocampal cortex correlates with behavioral performance in navigation (e.g., Igloi et al. 2010; Cornwell et al. 2008; Kong et al. 2016a). Given the complex nature, successful navigation likely requires multiple brain regions to work together (Wolbers and Hegarty 2010). A newly proposed model also suggests that a non-aggregate network process involving multiple interacting brain regions better characterizes the neural basis of spatial navigation (Ekstrom et al. 2014). Therefore, it is possible that the variability in human navigation may be better reflected by the underlying functional organization of the navigation network. A resting-state neuroimaging study has shown a positive correlation of navigation ability with the learning-induced changes in the functional connectivity between the parahippocampal cortex and the hippocampus (Wegman and Janzen 2011). Moreover, with spatial memory tasks, previous studies have shown that the dynamic interplay between multiple brain areas contributes to the behavioral outputs (Watrous et al. 2013; Arnold et al. 2014; Schedlbauer et al. 2014). More specifically, stronger global interaction during spatial memory retrieval is linked to successful retrieval (Watrous et al. 2013) and better performance (Schedlbauer et al. 2014). By combining resting-state fMRI with task fMRI, Arnold and colleagues (2014) for the first time, have revealed an association between global efficiency of the resting-state network composed of several brain regions engaged in orientation decision making and the behavioral performance in that task, providing a novel insight into the role of brain network variability in shaping one's ability to navigation the world (Arnold et al. 2014). However, this study mainly focused on brain regions engaged in a specific spatial task (i.e., the orientation decision task), and the brain-behavior correlation was based on a relatively small sample size $(N=13)$. Further research is required to better understand how brain regions, which are commonly activated in different navigation tasks, intrinsically interact with each other and how this intrinsic organization associates with navigation ability. To fill in this gap, in the present study, we aimed to construct a navigation network based on a neuroimaging meta-analysis of spatial navigation studies, and to explore the topological properties of navigation network and its behavioral correlates with a relatively large sample of subjects $(N=190)$.

To do this, we first defined the nodes of the navigation network (i.e., navigation-related cortical regions) with a large-scale fMRI meta-analysis using the Neurosynth (Yarkoni et al. 2011). Secondly, we constructed the navigation network of these functionally meaningful regions for each participant with the resting-state fMRI dataset in a large cohort of healthy young adults $(N=190)$. Third, we investigated three major topological properties of the navigation network using graph theory, including smallworldness, modularity, and hubs. Finally, we examined whether these topological properties were related to individual variability in navigation ability measured by the Santa Barbara Sense of Direction scale (SBSOD). We expected that the individual with more optimally organized navigation network (e.g., greater small-worldness and modularity) would possess better navigation ability.

\section{Materials and methods}

\section{Participants}

One hundred and ninety college students [ 117 females; mean age $=20.3$ years, standard deviation $(\mathrm{SD})=0.91$ years $]$ from Beijing Normal University (BNU), Beijing, China, participated in the study. The dataset was part of our ongoing project on bridging the brain, behaviors and genetics (http:// www.brainactivityatlas.org). All participants had normal or corrected-to-normal vision. The study was approved by the Institutional Review Board of BNU. Written informed consent was obtained from all participants before they took part in the experiment.

All participants $(N=190)$ underwent the fMRI scanning. No participant was excluded due to excessive head motion ( $2 \mathrm{~mm}$ in translation or 2 degree in rotation from the first volume in any axis) or visually detected registration errors (Zhen et al. 2015; Kong et al. 2016b). Most of these participants $(N=167 ; 104$ females; mean age $=20.2$ years, $\mathrm{SD}=0.90$ years) completed three behavioral assessments, including a standard questionnaire on spatial navigation performance in daily life, a computer test on small-scale spatial ability, and a Raven task for general ability (see below). 


\section{MRI scanning}

Scanning was conducted at BNU Imaging Center for Brain Research, Beijing, China, on a Siemens 3T scanner (MAGENTOM Trio, a Tim system) with a 12-channel phased-array head coil. The resting-state scan lasted $8 \mathrm{~min}$ and consisted of 240 contiguous echo-planar-imaging (EPI) volumes $\left(\mathrm{TR}=2000 \mathrm{~ms}\right.$; $\mathrm{TE}=30 \mathrm{~ms}$; flip angle $=90^{\circ}$; number $\quad$ of $\quad$ slices $=33 ; \quad$ matrix $=64 \times 64$; FOV $=200 \times 200 \mathrm{~mm}^{2} ; \quad$ acquisition voxel size $=3.125 \times 3.125 \times 3.6 \mathrm{~mm}^{3}$ ). During the scan, participants did not engage in any specific task and were instructed to relax and remain still with their eyes closed.

High-resolution T1-weighted images also were acquired with magnetization prepared gradient echo sequence (MPRAGE: $\quad$ TR/TE/TI $=2530 / 3.39 / 1100 \mathrm{~ms} ; \quad$ flip angle $=7^{\circ}$; matrix $=256 \times 256$ ) for spatial registration. One hundred and twenty-eight contiguous sagittal slices were obtained with $1 \times 1 \mathrm{~mm}^{2}$ in-plane resolution and $1.33-\mathrm{mm}$ slice thickness.

\section{Imaging data analysis}

Data preprocessing For each participant, image preprocessing was performed with FMRIB Software Library (FSL, http://www.fmrib.ox.ac.uk/fsl/). Preprocessing included head motion correction (by aligning each volume to the middle volume of the image with MCFLIRT), spatial smoothing (with a Gaussian kernel of 6-mm full-width halfmaximum), intensity normalization, and removal of linear trend. Next, a temporal band-pass filter $(0.01-0.1 \mathrm{~Hz})$ was applied with fslmaths to reduce low frequency drifts and high-frequency noise. Please note that the outputs of head motion correction were used to estimate individual's head motion during scanning with the averaged root-mean-square deviation (e.g., Jenkinson et al. 2002; Kong et al. 2014).

Registration of each participant's high-resolution anatomical image to a common stereotaxic space [the Montreal Neurological Institute (MNI) 152-brain template with a resolution of $2 \times 2 \times 2 \mathrm{~mm}^{3}$, MNI152] was accomplished using a two-step process (Andersson et al. 2007a). Firstly, a 12-degrees-of-freedom linear affine transformation was carried out with FLIRT (Jenkinson et al. 2002; Jenkinson and Smith 2001). Secondly, the registration was further refined with FNIRT nonlinear registration (Andersson et al. 2007b). Registration of each participant's functional images to the high-resolution anatomical images was carried out with FLIRT to produce a 6-degrees-of-freedom affine transformation matrix.

To eliminate physiological noise, such as fluctuations caused by motion or cardiac and respiratory cycles, nuisance signals were regressed out using the methods described in previous studies (Biswal et al. 2010; Fox et al. 2005). Nuisance regressors included averaged cerebrospinal fluid signal, averaged white matter signal, global signal averaged across the whole brain, six head realignment parameters obtained by rigid-body head motion correction, and the derivatives of each of these signals. The 4-D residual time series obtained after removing the nuisance covariates were registered to MNI152 standard space by applying the previously calculated transformation matrix. Moreover, to further rule out the possible influence of head motion during scanning (Power et al. 2012; Van Dijk et al. 2012; Zeng et al. 2014; Kong et al. 2014), individual's head motion was estimated from the fMRI data by integrating outputs of head motion correction.

\section{Navigation network construction}

A key issue in characterizing the human brain as a complex network is the construction of the connection matrix (Sporns et al. 2005). In this section, we applied a novel approach to define the nodes and edges in describing the navigation network.

Node definition To define nodes that are involved in spatial navigation, we conducted a neuroimaging meta-analysis on spatial navigation studies using the Neurosynth (neurosynth.org) (Yarkoni et al. 2011). Neurosynth uses textmining techniques to detect frequently used terms (as proxies for concepts of interest) in the neuroimaging literature: Terms that occur at a high frequency in a given study are associated with all activation coordinates in that publication, allowing for automated term-based meta-analysis. Despite the automaticity and the potentially high noise resulting from the association between term frequency and coordinate tables, this approach has been shown to be quite robust and reliable (e.g., Yarkoni et al. 2011; Helfinstein et al. 2014; Lebedev et al. 2014). The database was accessed on Nov 7 , 2014, searching for the feature "navigation" (55 studies with 2765 activations) (Fig. 1). The resulting forward inference map (i.e., the probability map that shows there would be activation in some specific brain regions given the presence of a particular term, e.g., 'navigation') was used in this study to cover regions that are relevant to navigation tasks. To control the false positive rate in the statistical map, a false discovery rate (FDR) threshold of 0.01 on a whole brain basis was used.

Then, the clusters were described by their corresponding anatomical labels in the automated anatomical labeling (AAL) atlas (Tzourio-Mazoyer et al. 2002) following the labeling protocol reported by Lebedev et al. (2014). We only included clusters larger than 100 voxels in this study as the nodes, which were further used for each individual's navigation network construction. 


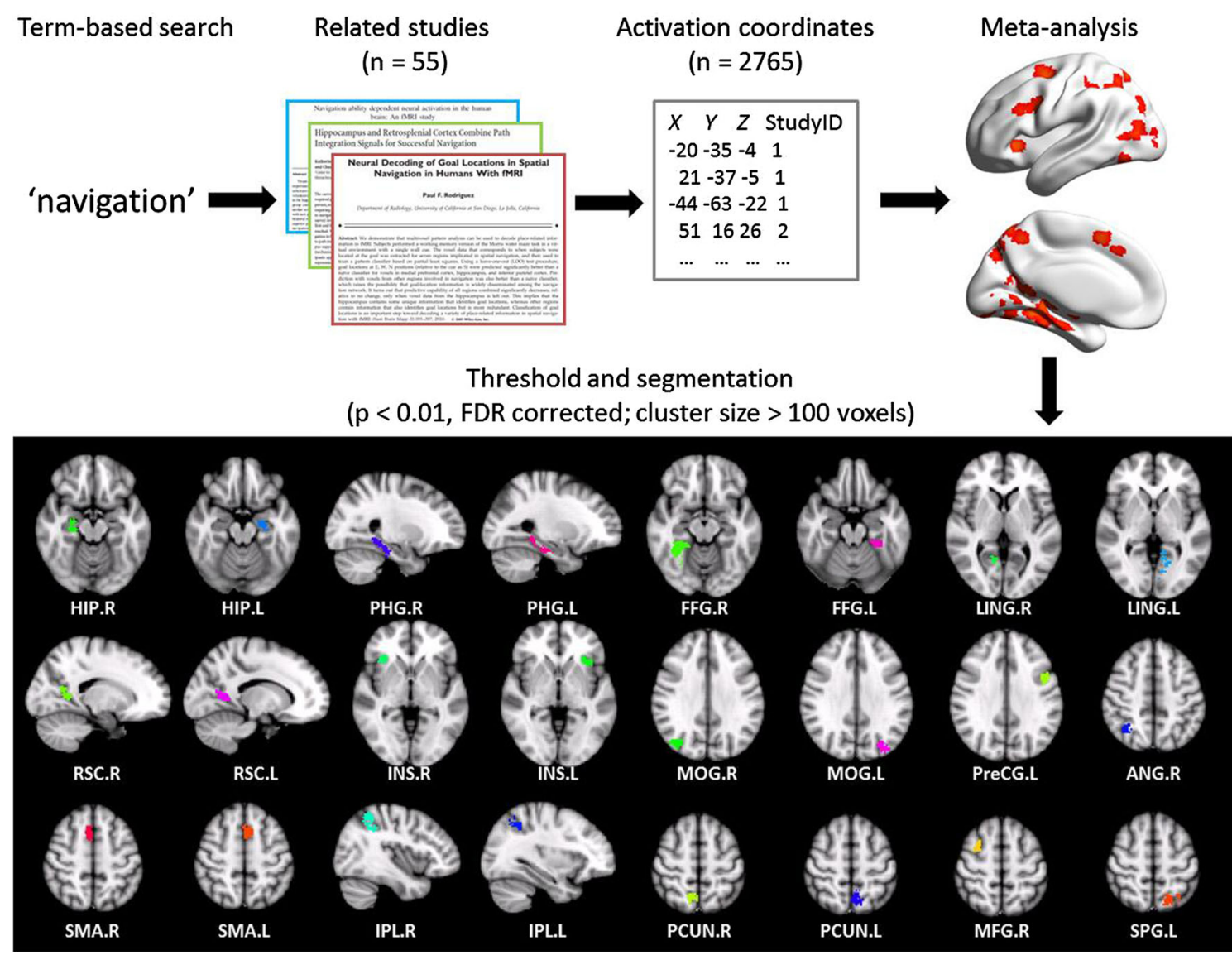
tion-associated ROIs. The neuroimaging meta-analysis was conducted using the Neurosynth (http://neurosynth.org) with the term "navigation". The profile included temporal, occipital, parietal and prefrontal
Fig. 1 General workflow for the automated identification of naviga-

regions ( $p<0.01$, FDR corrected). After removing clusters with relatively small sizes (i.e., less than 100 voxels), we obtained 24 ROIs for further network analyses. Node labels defined in Table 1

\section{Network analysis}

Edge definition After the preprocessing, a continuous time course for each ROI was extracted by averaging the time courses (from resting-state fMRI; 240 TRs; TR $=2000 \mathrm{~ms}$ ) of all voxels within that ROI. Thus, we obtained a time course consisting of 236 data points (removed first 4 time points) for each ROI and for each participant. Temporal correlation coefficients between the extracted time course from a given ROI and those from other ROIs were calculated to determine the strength of the connections between each pair of regions at rest. Correlation coefficients $(r)$ were transformed to Gaussian-distributed $z$ scores via Fisher's transformation to improve normality (Fox et al. 2006), resulting in a symmetric $N \times N Z$ value connection matrix (i.e., functional connectivity) for the navigation network of each participant ( $N$ represented the number of nodes). To remove spurious correlations, we set the functional connectivity with $p$ values higher than a statistical threshold $(p>0.05$, Bonferroni-corrected) to zero. Due to the ambiguous biological explanation of negative correlations (Fox et al. 2009; Murphy et al. 2009), we restricted our analyses to positive correlations.
For each navigation network, we focused on three most important principles of complex networks: small-worldness, modularity and hubs (Bullmore and Sporns 2009). In brief, small-worldness measures the extent to which a network allows highly efficient parallel information processing for a low wiring cost (Bassett and Bullmore 2006); modularity quantifies the decomposability of a network into modules (Meunier et al. 2009); and hubs are regions positioned to make big contributions to global network function (van den Heuvel and Sporns 2013). In the current study, all graph theoretical analyses were carried out on the weighted navigation network for each participant using GRETNA (Wang et al. 2015).

Small-worldness The small-world model is originally proposed by Watts and Strogatz (1998). Small-worldness was quantified using characteristic path length $\left(L_{\mathrm{p}}\right)$, and the clustering coefficient $\left(C_{\mathrm{p}}\right) . L_{\mathrm{p}}$ is defined as the average of the shortest path lengths between all pairs of nodes. 
Table 1 Spatial localization in MNI space coordinates and average $z$ scores for the sites in this meta-analysis

\begin{tabular}{|c|c|c|c|c|}
\hline Anatomical lable & Hemi. & Abbr. & Coordinates (MNI) & Avg. $Z$ score \\
\hline Precental gyrus & $\mathrm{L}$ & PreCG.L & $-47,6,33$ & 4.84 \\
\hline Middle frontal gyrus & $\mathrm{R}$ & MFG.R & $29,5,57$ & 4.81 \\
\hline \multirow[t]{2}{*}{ Supplementary motor area } & $\mathrm{L}$ & SMA.L & $-4,12,51$ & 5.17 \\
\hline & $\mathrm{R}$ & SMA.R & $6,12,52$ & 4.79 \\
\hline \multirow[t]{2}{*}{ Insula } & $\mathrm{L}$ & INS.L & $-33,23,-2$ & 4.89 \\
\hline & $\mathrm{R}$ & INS.R & $35,24,-3$ & 5.14 \\
\hline \multirow[t]{2}{*}{ Hippocampus } & $\mathrm{L}$ & HIP.L & $-23,-14,-18$ & 4.58 \\
\hline & $\mathrm{R}$ & HIP.R & $25,-27,-8$ & 4.27 \\
\hline \multirow[t]{2}{*}{ Parahippocampal gyrus } & $\mathrm{L}$ & PHG.L & $-25,-36,-13$ & 5.18 \\
\hline & $\mathrm{R}$ & PHG.R & $26,-34,-13$ & 5.64 \\
\hline \multirow[t]{2}{*}{ Retrosplenial cortex } & $\mathrm{L}$ & RSC.L & $-13,-57,6$ & 5.23 \\
\hline & $\mathrm{R}$ & RSC.R & $17,-57,11$ & 4.79 \\
\hline \multirow[t]{2}{*}{ Lingual gyrus } & $\mathrm{L}$ & LING.L & $-19,-83,-12$ & 4.80 \\
\hline & $\mathrm{R}$ & LING.R & $18,-48,-3$ & 4.60 \\
\hline \multirow[t]{2}{*}{ Middle occipital gyrus } & $\mathrm{L}$ & MOG.L & $-32,-81,30$ & 4.38 \\
\hline & $\mathrm{R}$ & MOG.R & $37,-76,27$ & 4.35 \\
\hline \multirow[t]{2}{*}{ Fusiform gyrus } & $\mathrm{L}$ & FFG.L & $-27,-41,-17$ & 4.55 \\
\hline & $\mathrm{R}$ & FFG.R & $32,-43,-16$ & 5.66 \\
\hline Superior parietal gyrus & $\mathrm{L}$ & SPG.L & $-18,-64,57$ & 4.67 \\
\hline \multirow[t]{2}{*}{ Inferior parietal gyri } & $\mathrm{L}$ & IPL.L & $-33,-56,46$ & 4.38 \\
\hline & $\mathrm{R}$ & IPL.R & $42,-47,47$ & 4.45 \\
\hline Angular gyrus & $\mathrm{R}$ & ANG.R & $34,-56,48$ & 4.29 \\
\hline \multirow[t]{2}{*}{ Precuneus } & $\mathrm{L}$ & PCUN.L & $-6,-64,52$ & 4.64 \\
\hline & $\mathrm{R}$ & PCUN.R & $8,-61,55$ & 4.54 \\
\hline
\end{tabular}

Smaller $L_{\mathrm{p}}$ indicates higher level of communication efficiency between nodes. $C_{\mathrm{p}}$ is defined as the fraction of the node's neighbors that are neighbors of each other, and reflects the prevalence of clustered connectivity around individual nodes. We normalized the $L_{\mathrm{p}}$ and $C_{\mathrm{p}}$ by dividing by the value for the same variable calculated from corresponding random networks. We generated these random networks $(n=1000)$ using Maslov's random rewiring algorithm (Maslov and Sneppen 2002), which preserves the same number of nodes, number of edges, and degree distribution as the real network. Small-worldness $(\delta)$ was computed as the ratio of the normalized $C_{\mathrm{p}}(\gamma)$ to the normalized $L_{\mathrm{p}}(\lambda)$ (Humphries and Gurney 2008). Thus, larger $\delta$ indicates relatively higher efficient parallel information processing at lower wiring cost. A small-world network is defined as a network with a summary smallworldness metric $\delta$ larger than 1 (Bullmore and Sporns 2009; Humphries and Gurney 2008).

Modularity Modularity is defined as the extent to which a network can be decomposed into sub-network (a.k.a. modules or communities) that are more connected within modules than between modules (Newman 2004). Modularity captures an important organizational principle critical to biological systems: integration within sub-systems allows efficient local processing, while sparse connections between sub-systems reduce the propagation of noise (Simon 1962). Thus, greater modularity characterizes more optimal system organization. In the present study, the modular architecture and modularity of navigation networks were estimated using a greedy optimization algorithm (Chen et al. 2008; Danon et al. 2006) as implemented in the GRETNA. This algorithm is to find a specific partition which yields the largest modularity. In practice, the value of the modularity of a network with a strong modular structure typically ranges from 0.3 to 0.7 (Newman and Girvan 2004). To estimate the robustness of the modular partition, we evaluated the significance of modularity of the navigation networks by comparing with that of 1000 node- and degree-matched random networks (He et al. 2009; Chen et al. 2008).

Hubs in the navigation network Important brain regions (i.e., hubs) often interact with many other regions in the complex network. In this study, we applied betweenness (Freeman 1977) to assess the importance of individual nodes. The betweenness of a node is defined as the number of shortest paths between pairs of other nodes that pass through the node (Freeman 1977), thus it captures the influence of a node over information flow between other nodes in the network. Navigation hubs were defined as 
nodes with a betweenness value higher than one SD above the corresponding average value in the navigation network. The results were visualized using BrainNet Viewer (Xia et al. 2013).

To investigate the specificity of the navigation network, we also constructed each individual's language networks using the same approach as above. For node definition, the feature "language comprehension" was used in the neuroimaging analysis, which included 76 studies with 2765 activations. Note that the sample sizes of both studies and activations were comparable to those for navigation network.

\section{Behavioral assessments}

Santa Barbara Sense of Direction scale (SBSOD) Navigation ability was measured using the SBSOD scale (Hegarty et al. 2002), which is a standard questionnaire on sense of direction in a large-scale environment, and is increasingly used as a reliable proxy for actual navigation ability (Janzen et al. 2008; Wegman and Janzen 2011). SBSOD consists of 15 items. Example items are "I very easily get lost in a new city" and "I can usually remember a new route after I have traveled it only once". Participants were instructed to indicate the extent to which they agreed or disagreed with each statement in a 5-point Likert-type scale. The total score was used to index one's navigation ability, with higher scores indicating better performance in daily navigation.

Previous studies have shown that people are aware of their own navigational ability (Kozlowski and Bryant 1977; Sholl 1988; Wolbers and Hegarty 2010), and the self-report measure from SBSOD was found to correlate with many objective measures on spatial navigation such as pointing errors in updating one's location in space while moving in the environment and pointing errors to unseen landmarks (Hegarty et al. 2002). Moreover, this scale has been increasingly used as a reliable proxy for real-world navigation performance in recent structural and functional neuroimaging literature (Janzen et al. 2008; Wegman and Janzen 2011; Wegman et al. 2014; Auger et al. 2012; Epstein et al. 2005). Therefore, SBSOD score, although based on participants' subjective experiences, is a valid index of the participants' real-world spatial navigation ability (see below for more discussion).

Mental rotation task (MRT) To measure individual's smallscale spatial ability, participants were administered the MRT (Shepard and Metzler 1971), consisting of 40 trials. Each trial started with a blank screen for $0.5 \mathrm{~s}$, followed by the first cube stimulus presented at the center of the screen. The three-dimensional asymmetrical assemblages of cube image were presented for $0.7 \mathrm{~s}$. After an inter stimulus interval (ISI) of $0.5 \mathrm{~s}$, the second stimulus appeared for the same duration as the first one, with the viewpoint being changed. Subjects were instructed to indicate whether the second stimulus was the first one rotated or a different stimulus as quickly as possible. Participants were given 3 min to finish all 40 trials, including 20 trials of 'rotated' condition and 20 of 'another' condition. The accuracy indexed each individual's mental rotation ability.

Raven's advanced progressive matrices (RAPM). To eliminate the possible influence of the general ability on the relationship between navigation ability and the organization of the navigation network, individual's general intelligence was measured using the standard RAPM (Raven 1995). The number of correct responses to the test items of RAPM was used to index intelligence for this study.

\section{Statistical analysis}

In addition to investigating the underlying organization properties (i.e., small-worldness, modularity, and hubs) of the navigation network, we explored the behavioral significance of these properties. Specifically, we related each of these measures to the variability in navigation ability with partial correlation analysis, controlling age and sex. Since multiple comparisons were performed in the analysis with nodal betweenness (number of ROIs $=24$ ), a significant threshold of FDR-corrected $q$ value of 0.05 was applied.

\section{Results}

\section{Construction of the navigation network}

We first constructed individual's navigation networks to investigate the underlying organization. Unlike previous network studies using an existing anatomical atlas or extra task fMRI for nodes definition, which is either functionally nonspecific or rather task dependent and time-consuming, we identified nodes involved in human spatial navigation from a neuroimaging meta-analysis on fMRI studies of navigation using the Neurosynth (Fig. 1; see "Materials and methods"). As expected, the resulting map consisted of medial and posterior temporal, parietal, and frontal regions, which have been commonly revealed by navigation studies (e.g., Ohnishi et al. 2006). Furthermore, 24 nodes were identified for navigation network construction, including the bilateral hippocampus (HIP.L/R), parahippocampal gyrus (PHG.L/R), retrosplenial complex (RSC.L/R; anterior part of the "calcarine fissure and surrounding cortex" in AAL), lingual gyrus (LING.L/R), middle occipital gyrus (MOG.L/G), fusiform gyrus (FFG.L/R), inferior parietal 
A

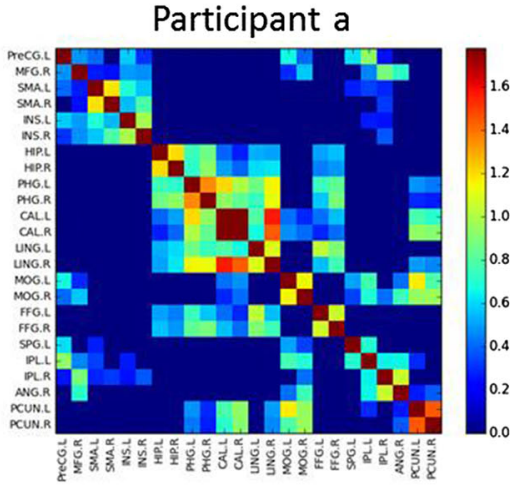

B

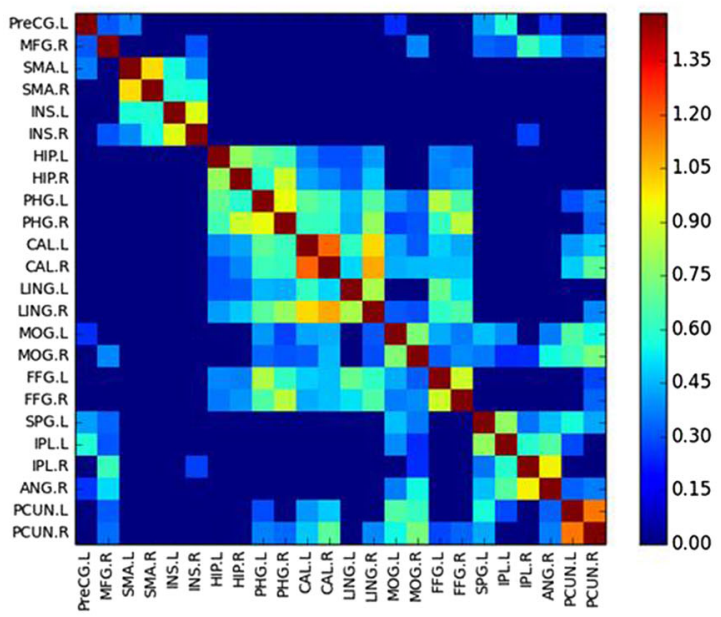

Participant b

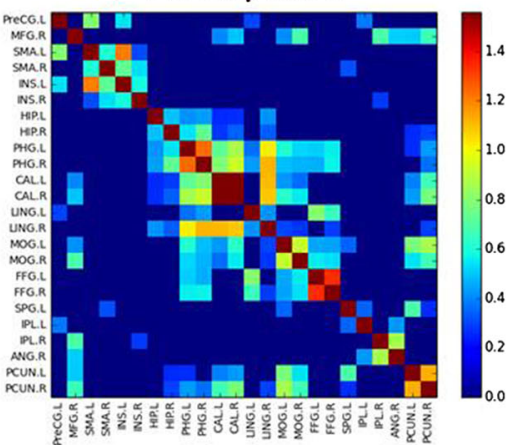

Participant c

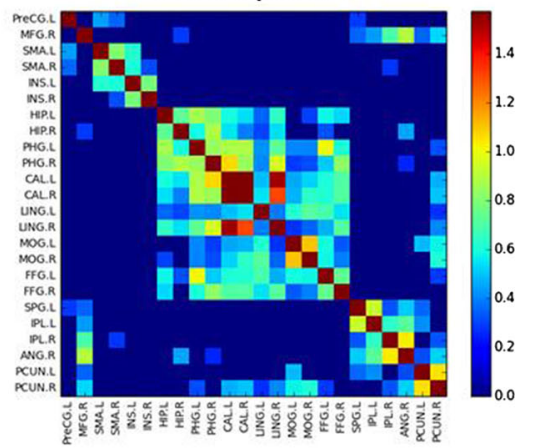

C

L
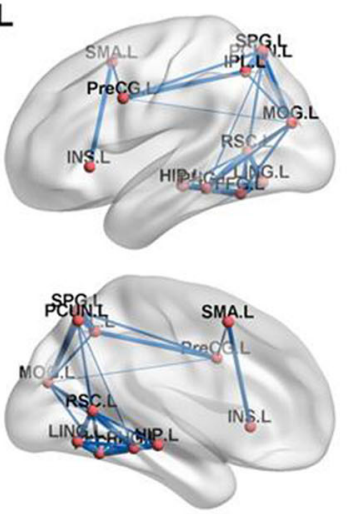

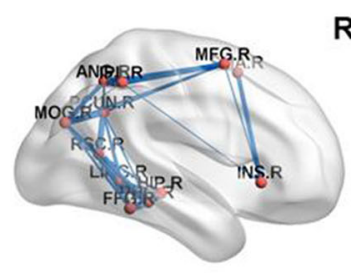

$\mathbf{R}$

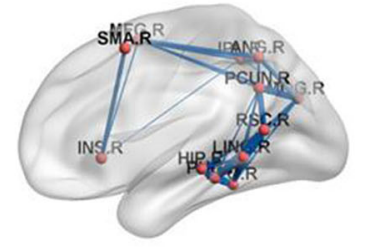

Fig. 2 a The navigation networks of 3 randomly selected participants. b The average of the navigation networks across the population. $\mathbf{c}$ The averaged navigation network visualized in 3D brain. Line thickness indicates strength of connection. Node labels defined in Table 1

gyri (IPL.L/R), insula (INS.L/R), precuneus (PCUN.L/R) and supplementary motor area (SMA.L/R), the left precentral gyrus (PreCG.L) and superior parietal gyrus (SPG.L), and the right middle frontal gyrus (MFG.R) and angular gyrus (ANG.R) (Table 1).

With these nodes, individual navigation network was constructed by calculating the functional connectivity matrix from the resting-state fMRI (see "Materials and methods"). Figure $2 \mathrm{a}$ shows the navigation networks from 3 randomly selected participants. In addition, a representative navigation network was obtained by averaging individual navigation networks across all the participants (Fig. 2b, c).

Next, we examined multiple topological parameters, including small-worldness and modularity of the navigation network, and nodal betweenness (for hub identification), as well as the behavioral correlates.

\section{Topological properties of the navigation networks and the behavioral relevance}

The small-worldness of the navigation network was estimated for each participant using graph theory (see
"Materials and methods"). Results showed that on average, the small-worldness of individual navigation networks was significantly larger than 1 (Mean $=1.36, \quad \mathrm{SD}=0.25$; $t(189)=73.58, p<0.001)$, suggesting a small-world organization of the navigation network. That is, in the healthy young adults, the intrinsic navigation networks are economical, tending to minimize wiring costs while supporting complex spatial navigation.

Next we used SBSOD to measure individuals' navigation ability. The results showed considerable individual differences in navigation ability (Table 2). Moreover, the Shapiro-Wilk normality test showed that the SBSOD scores were normally distributed $(W=0.99, p=0.223)$. Next, we examined whether the small-worldness of individual navigation network was related to individual differences in navigation ability. We found a significant correlation between navigation ability and small-worldness of the navigation network (Fig. 3; $r=0.18, p=0.021$; partial $r=0.18, p=0.022$ ), indicating that better navigation performance was associated with increased smallworldness of the navigation network. This association fits nicely with a general principle of brain organization that 
Table 2 Descriptive statistics of behavioral measures

\begin{tabular}{lcc}
\hline Variables & Mean & SD \\
\hline Age & 20.25 & 0.91 \\
SBSOD & 48.72 & 9.55 \\
RAPM & 25.54 & 4.20 \\
MRT & 0.66 & 0.09 \\
Head motion & 0.08 & 0.03 \\
\hline
\end{tabular}

Head motion was estimated from the resting-state fMRI data

$S D$ standard deviation, SBSOD Santa Barbara Sense of Direction scale, RAPM Raven's advanced progressive matrices, MRT mental rotation task

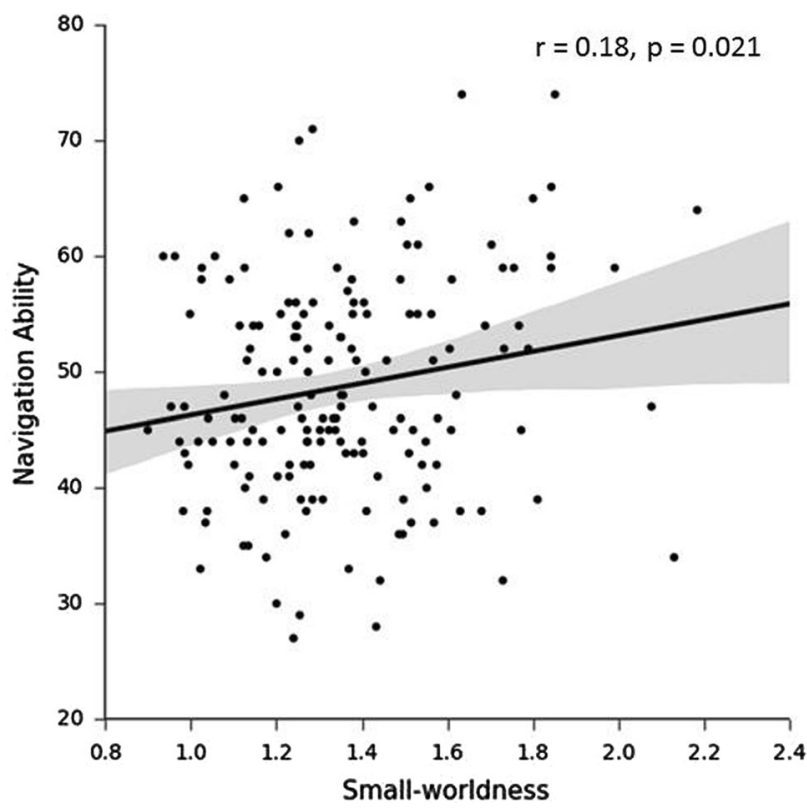

Fig. 3 Scatterplot represents the linear association between smallworldness of the navigation network and navigation performance. Shaded regions depict $95 \%$ confidence intervals

the degree to which the brain networks is optimized relates to the effective cognitive functioning (e.g., spatial navigation) of the individual (Stevens et al. 2012).

Based on previous studies, a small-world network is organized into modules of densely interconnected nodes, but among modules only sparsely interconnected (Rubinov and Sporns 2010; Newman 2006). Next we investigated the intrinsic modular architecture of the navigation network, and explored the relationship between modularity of individual's network and individual differences in navigation ability.

To reliably reveal the modular structure of navigation network, we first conducted the modularity analysis on the representative navigation network which was obtained by averaging across all participants (Fig. 2b, c). Results showed that the navigation network was separated into three modules (Fig. 4a): a medial temporal module including the bilateral HIP, RSC, PHG, LING, and FFG; a posterior-dorsal module including the bilateral MOG, IPL, PCUN, and right MFG, ANG, and left PreCG, and SPL; and an anterior module including the bilateral SMA and INS. Based on previous studies, the medial temporal module might serve as a core network which was primarily responsible for visual spatial processing to generate the spatial representation of the environment (Epstein and Kanwisher 1998; Marchette et al. 2014; O'Keefe and Dostrovsky 1971); the posterior-dorsal and anterior modules, as two components of an extended network, might involve spatial imagery and orientation decision making (Mellet et al. 1995; Arnold et al. 2014; Harvey et al. 2012), and somatosensory information processing (Mesulam and Mufson 1982; Ghaem et al. 1997; Jahn et al. 2004) during navigation, respectively (see below for discussion). Moreover, the statistically significant modularity $(Q=0.38$, $Z$ score $=14.36)$ of the navigation network implies that the underlying modular architecture arises from the specific interplay among distributed regions.

Finally, we calculated modularity for each individual's navigation network, and then related it to individual's navigation ability. Similar to the averaged navigation network, individual's network also showed a modular structure $($ Mean $=0.39, \mathrm{SD}=0.06 ; Z$ score $=[2.83,18.90])$. More importantly, we found the network modularity was significantly correlated with individual's navigation ability (Fig. 4b; $\quad r=0.24, \quad p=0.002 ;$ partial $r=0.23$, $p=0.003)$. Specifically, individuals who possess stronger modularity (i.e., stronger intra-modular connections and weaker inter-modular connections) showed better navigation ability, suggesting that increased modularity of the navigation network might facilitate one's navigation performance in daily life. This might be related to the modular structure of the navigation network that allows more efficient local processing within a module and reduces the propagation of noise between modules (Simon 1962).

Having highly connected hubs is another feature of complex brain networks. Next, we explored the hubs within the navigation network, and investigated their behavioral relevance. In the present study, betweenness was used to index the importance of the node in the navigation network.

To reliably identify hubs in the navigation network, we first assessed betweenness for each node based on the representative navigation network (Fig. 2b, c). The spatial distribution of nodes is shown in Fig. 5a, with larger spheres representing higher betweenness values, and the top 10 nodes are mainly located in the core network for spatial navigation and the parietal extended network (Fig. 5b). Furthermore, according to the criteria for 
A

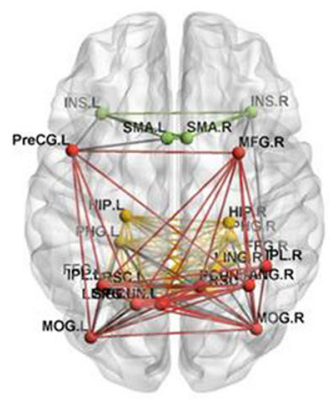

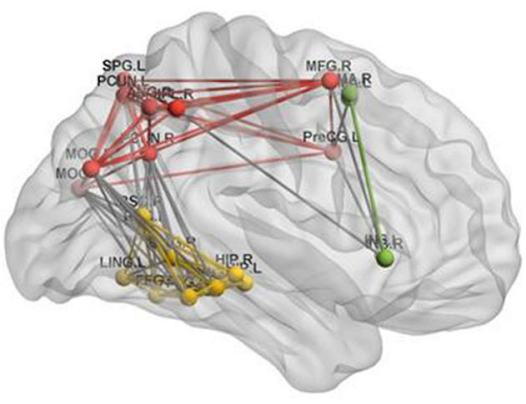

B

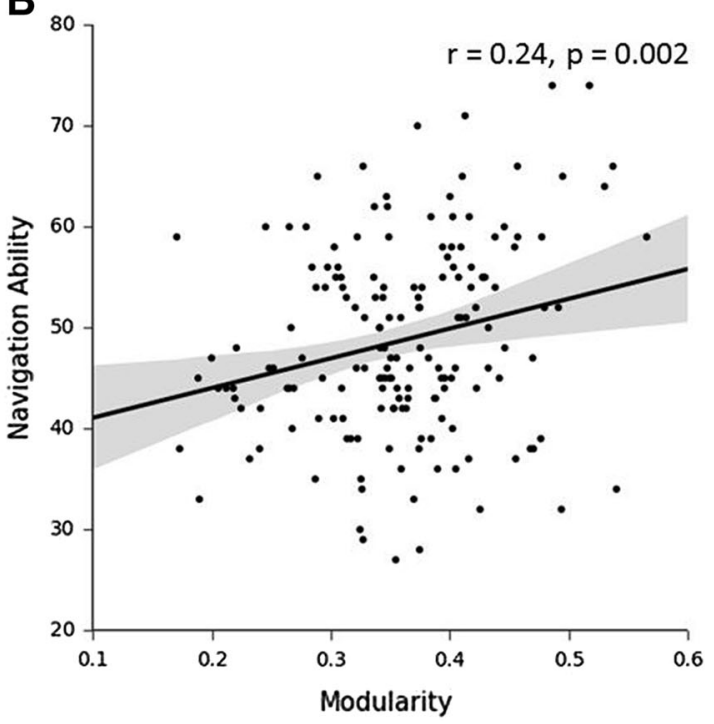

Fig. 4 a Modularity structure of the navigation network. Node color represents affiliation of each region as defined by graph analytic modularity analysis (yellow, red, and green). b Scatterplot represents the linear association between modularity of the navigation network and navigation performance. Shaded regions depict $95 \%$ confidence intervals
A

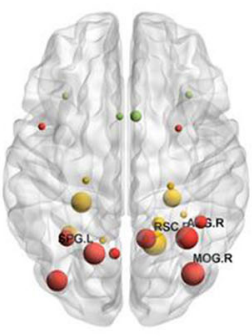

B
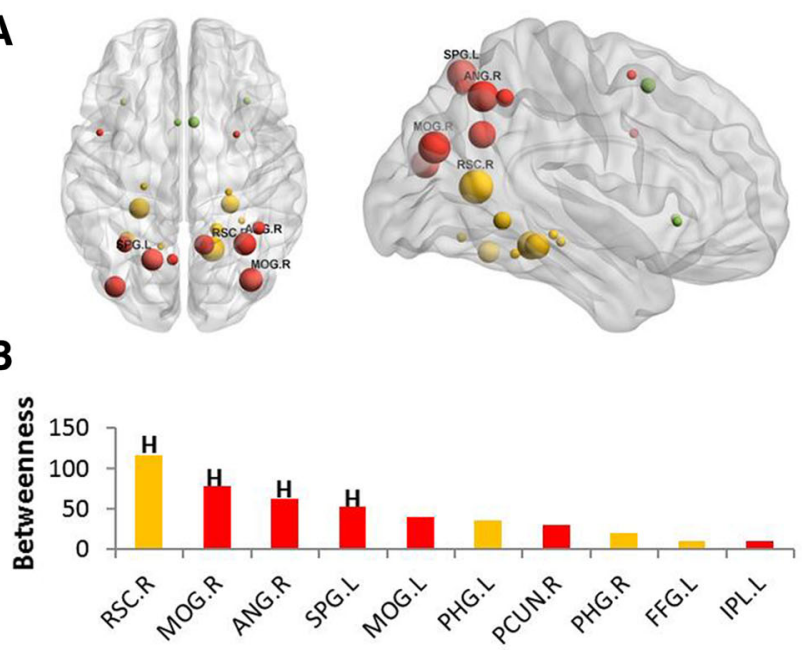

C

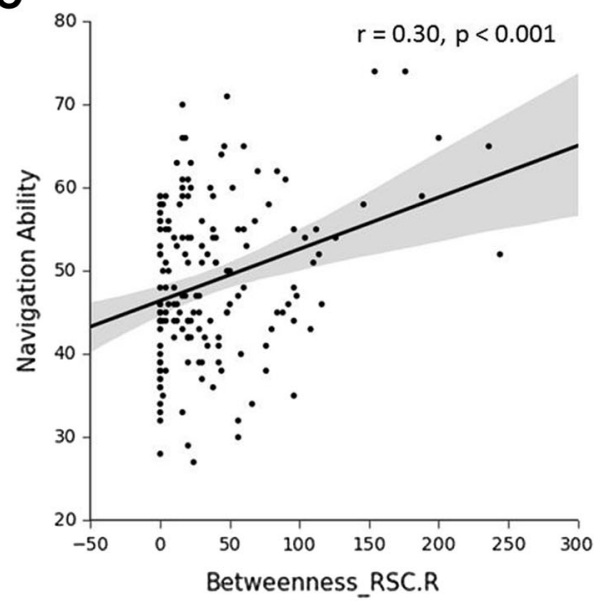

Fig. 5 a The spatial distribution of nodal betweenness for the navigation network, with larger spheres representing a higher betweenness values. Four hubs were identified including the RSC.R, MOG.R, ANG.R, and SPG.L. b The top 10 nodes of betweenness in the navigation network. Nodes are ordered from the largest betweenness (left) to the smaller (right). Node color represents different

identifying hubs (see "Materials and methods"), 4 hubs of this network were identified, including the right RSC, MOG, and ANG, and left SPG.

Next, we calculated betweenness for each node based on individual's navigation network, and examined whether the individual differences in nodal betweenness would predict variability in navigation ability. We found that, after correction of multiple comparisons, the betweenness of the modules, and $\mathbf{H}$ indicates hubs identified in the present study. c Scatter plot represents the significant correlation between the betweenness of the right RSC and navigation performance $(p<0.05$, FDR corrected). Shaded regions depict $95 \%$ confidence intervals. Node labels defined in Table 1

right RSC showed a positive correlation with individual's navigation ability (Fig. 5c; $r=0.30, p<0.001$; partial $r=0.29, p<0.001$; corrected $p<0.05)$. Given the nonnormality of the betweenness $(W=0.79, p<0.001)$, Spearman's correlation was used to verify the correlation. Results showed that the observed correlation remained significant $(\rho=0.23, p=0.003)$. These results suggest a critical role of the right RSC, as one of the hubs in the 
navigation network, in determining human navigation ability.

\section{Navigation-specific nature of the findings}

Given that the nodes used in this work have been implicated in navigation, we hypothesized that the associations between the navigation network and behavioral performance would possess a navigation-specific nature. To test this hypothesis, we conducted the following analyses.

First, to rule out the possibility that general ability contributed to the observed associations, we measured individual's general intelligence using the standard RAPM (Raven 1995) and found that neither the navigation ability nor the graph-theoretic measures of navigation networks showed significant association with general ability (i.e., intelligence) (navigation ability: $r=0.07, p=0.366$; small-worldness: $r=0.12, p=0.126$; modularity: $r<0.01, p=0.978$; betweenness of the right RSC: $r=-0.10, p=0.897)$. In addition, when the general intelligence was controlled, the observed associations between graph-theoretic measures of the navigation networks and individual variability in navigation ability remained significant (small-worldness: $r=0.18, p=0.023$; modularity: $r=0.21, p=0.009$; betweenness of the right RSC: $r=0.28, p<0.001$ ).

Second, we further investigated the specificity of the observed associations by measuring individual's smallscale spatial ability with MRT. As expected, we found no significant association between small-scale spatial ability and the graphic metrics of navigation networks (smallworldness: $r=0.07, p=0.330$; modularity: $r=0.04$, $p=0.574$; betweenness of the right RSC: $r=0.10$, $p=0.173$ ), suggesting that the associations were specific to large-scale spatial ability (i.e., navigation ability).

Third, we ran another control analysis to examine whether the observed navigation-related correlations were specific to the navigation network. With the same approach as for the navigation networks, we identified 15 nodes, including the left inferior frontal gyrus, precentral gyrus and superior and middle temporal gyrus, which have been commonly implicated in language comprehension (e.g., Tyler and Marslen-Wilson 2008; Fonteneau et al. 2015). After obtaining the measures for topological properties of the language networks, we related them to individual differences in navigation ability. As expected, we found no significant correlation with any of these topological measures of the language networks ( $p s>0.05$, uncorrected). These results suggested the observed correlations were unique to navigation network, which further supported the navigation-specific nature of our main findings.

Finally, the neuroimaging community has become increasingly concerned about the confounding effect of inscanner head motion on fMRI data (e.g., Power et al. 2012;
Van Dijk et al. 2012). Further, head motion has also been shown reflecting reliable traits (e.g., Kong et al. 2014; Zeng et al. 2014). To rule out the possible influence of head motion on the observed associations, we estimated participants' head motion from the fMRI data and we found that after controlling for head motion, the brain-behavior associations remained significant (small-worldness: $r=0.18, p=0.018$; modularity: $r=0.22, p=0.005$; betweenness of the right RSC: $r=0.29, p<0.001$ ).

Taken together, the navigation network showed robust associations with individual's perceived navigation ability in daily life, suggesting a navigation-specific nature of the observed brain-behavior associations.

\section{Discussion}

In this study, we first constructed individual's navigation network by combining a neuroimaging meta-analysis and the resting-state fMRI. Then, multiple topological properties, including small-worldness, modularity and nodal betweenness, were investigated with graphical theory. Finally, these graph-theoretic properties were related to each individual's navigation ability. The main findings of our study are: (1) navigation networks showed small-world and modular organization; (2) better navigation performance was associated with increased small-worldness and modularity; (3) the right RSC was revealed to be one of the hubs in the network; and (4) betweenness of the right RSC showed positive association with individual's navigation ability.

Human navigation is a complex process, and requires collaboration of multiple brain regions. Therefore, efficient information transfer and minimal wiring costs among these regions would be important for behaviors (i.e., spatial navigation), while insufficient communication would lead to poor performance (Stevens et al. 2012; Stam and Reijneveld 2007). Our findings that better navigation ability was associated with both small-worldness and modularity of the navigation network nicely fit these predictions, suggesting that efficient neural processing underlying flexible navigation may involve distributing cognitive load across different brain regions, rather than placing too much burden on any single network element (e.g., hippocampus). This is in line with a newly proposed model that a nonaggregate network process involving multiple interacting brain regions, including hippocampus and extra-hippocampal regions, better characters the neural basis for spatial navigation (Ekstrom et al. 2014). Moreover, the present study was based on a novel protocol to define nodes consistently involved in various navigation tasks (see below for more discussion), which would facilitate the construction of a more comprehensive model of the 
navigation network. By combining resting-state fMRI in a large cohort of young adults, the present study extended previous findings of the network-level dynamics based on specific spatial tasks and its contribution to behavioral performance (Watrous et al. 2013; Arnold et al. 2014; Schedlbauer et al. 2014). Taken together, our findings provide one of the first evidence that multiple distributed brain regions intrinsically work together as a network underlying the individual differences in navigation ability.

Intriguingly, in the present study, we found three relatively independent modules in the navigation network through synchronized neural fluctuations under a resting state: one as the core network for spatial navigation consisting of the medial temporal areas, and two as parts of the extended network for spatial navigation mainly located in the parietal and frontal regions, respectively. Specifically, the core network included regions like the PHG, RSC, and HIP, which are primarily responsible for three key components for successful navigation: visual scene processing (Epstein and Kanwisher 1998), spatial orientation (Marchette et al. 2014; Kim et al. 2015), cognitive map (O'Keefe and Dostrovsky 1971; Ekstrom et al. 2003), respectively. Both neuroimaging and neuropsychological studies have suggested the critical behavioral importance of the core network. For example, variations in structural and/or functional measures of these regions have been found to correlate with individual differences in navigation performance (Maguire et al. 2000; Epstein et al. 2005). Lesions in these regions (i.e., PHG and RSC) indeed impair patients' navigation abilities (Epstein et al. 2001; Mendez and Cherrier 2003; Takahashi et al. 1997). Unlike the core network, little is known about the precise functions of the extended network in spatial navigation, though considerable activation has been shown to be related to spatial navigation. According to previous studies, the parietal module may be related to spatial imagery and orientation decision making (Mellet et al. 1995; Arnold et al. 2014; Harvey et al. 2012), while the frontal module (i.e., SMA and INS) may be related to somatosensory information processing during navigation (Mesulam and Mufson 1982; Ghaem et al. 1997; Jahn et al. 2004). Note that the abovementioned behavioral significance is speculative, in particular for the extended network, and largely based on reverse inference of fMRI data (Poldrack 2011), and future studies on the specific functions of these relatively independent modules are needed. Another intriguing finding is that we found that a more modular topology of the navigation network was related to better navigation performance in daily life. While the higher modularized architecture could reflect various aspects of intrinsically organization of the navigation network including the balance of functional segregation and integration (He et al. 2009), our finding are not in contradiction with previous findings with network dynamics that global connectivity positively correlates with individual's behavioral performance (e.g., Schedlbauer et al. 2014). This difference might be due to the different approach for navigation network construction in the present study. For example, unlike Schedlbauer et al. (2014) and Arnold et al. (2014), we defined the nodes based on a large-scale neuroimaging meta-analysis; in addition, we estimated the connections based on resting-state fMRI, rather than task fMRI used in Schedlbauer et al. (2014). Further studies with fMRI when performing navigation tasks would provide more insights into the modular organization of navigation network and its dynamics.

In addition to modularity, having highly connected hubs is another topological feature of small-world networks. We found that the right RSC acted as one of the hubs in the navigation network, and that the betweenness of the RSC showed significant correlation with better navigation ability, suggesting a critical role of this region in modulating the dynamic information processing within the whole navigation network. In previous work, the RSC has been highlighted as an important area for navigation and route learning (Maguire et al. 1998; Maguire 2001; Cain et al. 2006; Cooper and Mizumori 2001; O'Craven and Kanwisher 2000). For instance, recent studies suggests that the RSC anchors our sense of direction to local environment (Marchette et al. 2014), and that an alteration of the RSC's functional properties may serve as the neural basis for developmental topographic disorientation (DTD) (Kim et al. 2015). Previous neuropsychological findings also showed that patients with damage in the RSC are unable to describe the relationship between locations and exhibited spatial navigation impairment (Takahashi et al. 1997; Aguirre and D'Esposito 1999; Valenstein et al. 1987; Vann et al. 2009). Furthermore, neuroimaging studies have suggested that the RSC involves in integration of spatial information for navigation (Epstein 2008; Mullally and Maguire 2011; Park and Chun 2009). The integration role may be contributed by the reciprocal connections of this region with multiple regions across the brain, such as the prefrontal and medial temporal regions (Vann et al. 2009). Thus, previous computational models of spatial memory (Byrne et al. 2007; Burgess et al. 2001; Vann et al. 2009) also consider the RSC as a key member of a core network of brain regions that underpins spatial memory and navigation. Specifically, the spatial information of location and environmental layout extracted by the hippocampus and parahippocampal cortex is hypothesized in the models to be used by the RSC, which translates allocentric information and egocentric information (Spiers and Maguire 2006). The output of this integrative processing in the RSC is then fed forward to regions in the precentral gyrus, which has been suggested to translate spatial representation into 
motor intentions in navigation (Ikkai and Curtis 2011). Taken together, our findings confirmed previous reports, were in line with previous computational models, and provided the first evidence of the critical role of the RSC in navigation from a holistic perspective of complex networks.

Moreover, in the brain-behavior correlation analyses, our results suggested that the degree to which the navigation network was optimally organized (e.g., greater smallworldness and modularity) was predictive of individual's navigation performance. Given the fact that the navigation networks were constructed with resting-state signal, how does intrinsic functional organization translate to behavioral variability? Small-worldness measures the parallel information processing efficiency, while modularity measures the level of integration and segregation across subnetworks. Previous resting-state studies have shown that both of them are related to the effective cognitive functioning, including working memory (Stevens et al. 2012) and normal aging (Onoda and Yamaguchi 2013). Our results suggested that the intrinsic functional organization of the navigation network modulated the degree to which an individual was able to intrinsically coordinate and synchronize the neural activity while navigating the world in daily life, which ultimately related to their navigation performance and perceived navigation ability. More importantly, we found that multiple topological properties, including small-worldness and modularity, of the navigation networks were only predictive of navigation ability, but not of small-scale spatial ability or general intelligence. Moreover, we did not find any significant correlation between a network not involved in navigation (i.e., language network) and navigation ability. All these results suggested that the observed brain-behavior correlations based on the resting-state network that was composed of navigation-relevant regions were specific to spatial navigation.

It is noteworthy that, in this study, we employed a novel protocol to define nodes for investigating the behavioral relevance of large-scale brain networks. Identification of reliable, reproducible, and functionally meaningful ROIs is critically important for successful mapping of brain networks for specific functions. Therefore, a fundamental question was raised when attempting to investigate the behavioral significance of specific brain networks: how to define the appropriate ROIs? The majority of previous brain network studies for individual differences (e.g., Li et al. 2009; Sala-Llonch et al. 2014; Breckel et al. 2013) have been based on predefined ROIs in a template brain or manual labeling by experts based on a priori knowledge. However, these approaches have limitations. For example, manual labeling is vulnerable to inter-expert and intra-expert variation and its reproducibility may be low (Sobel et al. 1993). More importantly, both approaches are usually based on anatomical information alone and segmentations of regions are functionally nonspecific. Task fMRI provides a benchmark approach for identifying functionally specific ROIs (e.g., scene-specific regions), but for investigating individual differences in complex behaviors (e.g., spatial navigation), this approach would be largely task dependent and time-consuming. Thus, instead of using anatomical segmentations or extra task fMRI for nodes definition, we defined the nodes relevant to spatial navigation using a large-scale neuroimaging meta-analysis with the Neurosynth. This tool has been suggested to be quite robust and reliable to define functionally meaningful nodes (FMNs) (e.g., Yarkoni et al. 2011; Helfinstein et al. 2014; Lebedev et al. 2014). On the one hand, given that the FMNs used in this study were not task-specific, these results advance previous research focusing on specific navigation task (Arnold et al. 2014) and allow a better understanding of the individual differences in navigation ability. On the other hand, the Neurosynth provides an automated synthesis method for large-scale neuroimaging studies (Yarkoni et al. 2011), allowing us to objectively identify FMNs for specific psychological features (e.g., navigation, executive control, and emotion). This method is publicly available and easy to use. Since the nodes identified with this method have widely been implicated in the specific cognition of interest, it would be expected to enhance the statistical power for the following brain-behavior correlation analysis. Thus, with these functionally meaningful regions and the large neuroimaging and behavioral dataset, the new framework used in this study would provide new opportunities for better understanding the brain-behavior associations from a complex network perspective.

The present study has some limitations. Most notably, the participants' navigational ability was assessed with a self-reported questionnaire (i.e., SBSOD), and the effect sizes of SBSOD in relation to behavioral performance in virtual and real-world environments appears to be relatively small (Arnold et al. 2013; Wolbers and Hegarty 2010). For example, the effect sizes for correlations with SBSOD scores were 0.18 for both the formation and use of cognitive maps (Arnold et al. 2013), similar to those observed in the present study, especially for small-worldness (i.e., 0.18). Thus, it is unclear whether these findings of behavioral relevance could translate to behavioral performance in different navigation tasks. But in the aspect of behavioral relevance, the present work extended the existing literature to show that self-perceived navigation ability correlated with the intrinsic topological properties of navigation networks. Nevertheless, future studies with different objective tasks for spatial navigation will provide more insights into the behavioral relevance of navigation 
networks. Another intriguing issue is that we found that quite a few nodes (e.g., HIP, PCUN, MFG, and SPG) in the navigation network are also part of a whole-brain 'richclub' network (van den Heuvel and Sporns 2011); thus the findings of behavioral relevance might reflect a relationship with general network properties across the entire brain. However, this would not be the case. First, the overlap is only partial, with several navigation network regions (e.g., PHG, RSC, PreCG, and MOG) not found among wholebrain rich-club region (van den Heuvel and Sporns 2011). Moreover, we did not find significant association between navigation network properties and general ability (which is reflected by the whole-brain general properties $\mathrm{Li}$ et al. 2009; van den Heuvel et al. 2009), and the observed associations between navigation network properties and navigation ability remained when the general ability was controlled. Finally, more importantly perhaps, we found that the navigation ability showed significant correlations with navigation network, but not with a network not involved in navigation (e.g., language network), which provided further evidence for the unique contribution of navigation network to navigation behaviors. Taken together, these findings suggested the navigation-specific nature of the observed brain-behavior associations.

In sum, we investigated the large-scale intrinsic organization of the navigation network in human brain and linked multiple optimal organizational properties of the networks to better navigation ability. These findings together provide a new insight into the neural basis of individual differences in navigation, and provide direct leads to addressing the precise relationship between brain organization and navigation. For example, further research is needed to understand how genetic and environmental factors contribute to the development of the navigation network. A further topic of interest concerns the dynamics of the navigation network: it is possible that the organization of the functional navigation networks could be modified during different navigation tasks. Although the brain's functional network architecture during performing tasks is shaped primarily by intrinsic network architecture, tasks also modify the network architecture (e.g., Cole et al. 2014). For example, we found that the RSC, a hub identified in the navigation network using resting-state fMRI, has not been reported acting as a hub for functional interactions during specific spatial tasks (Schedlbauer et al. 2014; Watrous et al. 2013), which might be due to different task demands (and different approaches for node definitions). Thus, our study invites further research into how spatial tasks modulate the functional configuration of navigation networks. Last, future investigations from the network perspective would help us understand how the navigation network relates to the behavioral impairment in the aging and psychiatric patients, such as mild cognitive impairment (MCI) and Alzheimer's disease (AD), and further understand the underlying neural mechanisms.

Acknowledgments This study was funded by the National Natural Science Foundation of China (31230031, 31221003, and 31470055), the National Basic Research Program of China (2014CB846103), National Social Science Foundation of China (13\&ZD073, 14ZDB160) and Changjiang Scholars Programme of China.

\section{References}

Aguirre GK, D'Esposito M (1999) Topographical disorientation: a synthesis and taxonomy. Brain 122(Pt 9):1613-1628

Andersson JLR, Jenkinson M, Smith S (2007a) Non-linear optimisation. FMRIB Technical report TR0JA2. FMRIB Centre, Oxford, United Kingdom. http://www.fmrib.ox.ac.uk/analysis/techrep

Andersson JLR, Jenkinson M, Smith S (2007b) Non-linear registration, aka Spatial normalisation. FMRIB Technical report TR0JA2. FMRIB Centre, Oxford, United Kingdom. http:// www.fmrib.ox.ac.uk/analysis/techrep

Arnold AE, Burles F, Krivoruchko T, Liu I, Rey CD, Levy RM, Iaria $\mathrm{G}$ (2013) Cognitive mapping in humans and its relationship to other orientation skills. Exp Brain Res 224(3):359-372. doi:10. 1007/s00221-012-3316-0

Arnold AE, Protzner AB, Bray S, Levy RM, Iaria G (2014) Neural network configuration and efficiency underlies individual differences in spatial orientation ability. J Cogn Neurosci 26(2):380-394. doi:10.1162/jocn_a_00491

Auger SD, Mullally SL, Maguire EA (2012) Retrosplenial cortex codes for permanent landmarks. PLoS One 7(8):e43620. doi:10. 1371/journal.pone.0043620

Bassett DS, Bullmore E (2006) Small-world brain networks. Neuroscientist 12(6):512-523. doi:10.1177/1073858406293182

Biswal BB, Mennes M, Zuo XN, Gohel S, Kelly C, Smith SM, Beckmann CF, Adelstein JS, Buckner RL, Colcombe S, Dogonowski AM, Ernst M, Fair D, Hampson M, Hoptman MJ, Hyde JS, Kiviniemi VJ, Kotter R, Li SJ, Lin CP, Lowe MJ, Mackay C, Madden DJ, Madsen KH, Margulies DS, Mayberg HS, McMahon K, Monk CS, Mostofsky SH, Nagel BJ, Pekar JJ, Peltier SJ, Petersen SE, Riedl V, Rombouts SA, Rypma B, Schlaggar BL, Schmidt S, Seidler RD, Siegle GJ, Sorg C, Teng GJ, Veijola J, Villringer A, Walter M, Wang L, Weng XC, Whitfield-Gabrieli S, Williamson $\mathrm{P}$, Windischberger C, Zang YF, Zhang HY, Castellanos FX, Milham MP (2010) Toward discovery science of human brain function. Proc Natl Acad Sci USA 107(10):4734-4739. doi:10.1073/pnas.0911855107

Breckel TP, Thiel CM, Bullmore ET, Zalesky A, Patel AX, Giessing C (2013) Long-term effects of attentional performance on functional brain network topology. PLoS One 8(9):e74125. doi:10.1371/journal.pone.0074125

Brown TI, Whiteman AS, Aselcioglu I, Stern CE (2014) Structural differences in hippocampal and prefrontal gray matter volume support flexible context-dependent navigation ability. J Neurosci 34(6):2314-2320. doi:10.1523/JNEUROSCI.2202-13.2014

Bullmore E, Sporns O (2009) Complex brain networks: graph theoretical analysis of structural and functional systems. Nat Rev Neurosci 10(3):186-198. doi:10.1038/nrn2575

Burgess N, Maguire EA, Spiers HJ, O'Keefe J (2001) A temporoparietal and prefrontal network for retrieving the spatial context of lifelike events. Neuroimage 14(2):439-453. doi:10.1006/nimg. 2001.0806

Byrne P, Becker S, Burgess N (2007) Remembering the past and imagining the future: a neural model of spatial memory and 
imagery. Psychol Rev 114(2):340-375. doi:10.1037/0033-295X. 114.2.340

Cain DP, Humpartzoomian R, Boon F (2006) Retrosplenial cortex lesions impair water maze strategies learning or spatial place learning depending on prior experience of the rat. Behav Brain Res 170(2):316-325. doi:10.1016/j.bbr.2006.03.003

Chen ZJ, He Y, Rosa-Neto P, Germann J, Evans AC (2008) Revealing modular architecture of human brain structural networks by using cortical thickness from MRI. Cereb Cortex 18(10):2374-2381. doi:10.1093/cercor/bhn003

Ciaramelli E (2008) The role of ventromedial prefrontal cortex in navigation: a case of impaired wayfinding and rehabilitation. Neuropsychologia 46(7):2099-2105. doi:10.1016/j.neuropsycho logia.2007.11.029

Cole MW, Bassett DS, Power JD, Braver TS, Petersen SE (2014) Intrinsic and task-evoked network architectures of the human brain. Neuron 83(1):238-251. doi:10.1016/j.neuron.2014.05.014

Cooper BG, Mizumori SJ (2001) Temporary inactivation of the retrosplenial cortex causes a transient reorganization of spatial coding in the hippocampus. J Neurosci 21(11):3986-4001 (pii: 21/11/3986)

Cornwell BR, Johnson LL, Holroyd T, Carver FW, Grillon C (2008) Human hippocampal and parahippocampal theta during goaldirected spatial navigation predicts performance on a virtual Morris water maze. J Neurosci 28(23):5983-5990. doi:10.1523/ JNEUROSCI.5001-07.2008

Danon L, Diaz-Guilera A, Arenas A (2006) Effect of size heterogeneity on community identification in complex networks. J Stat Mech Theory Exp 2006:P11010

Ekstrom AD, Kahana MJ, Caplan JB, Fields TA, Isham EA, Newman EL, Fried I (2003) Cellular networks underlying human spatial navigation. Nature 425(6954):184-188. doi:10.1038/ nature 01964

Ekstrom AD, Arnold AE, Iaria G (2014) A critical review of the allocentric spatial representation and its neural underpinnings: toward a network-based perspective. Front Hum Neurosci 8:803. doi:10.3389/fnhum.2014.00803

Epstein RA (2008) Parahippocampal and retrosplenial contributions to human spatial navigation. Trends Cogn Sci 12(10):388-396. doi:10.1016/j.tics.2008.07.004

Epstein R, Kanwisher N (1998) A cortical representation of the local visual environment. Nature 392(6676):598-601. doi:10.1038/ 33402

Epstein R, Harris A, Stanley D, Kanwisher N (1999) The parahippocampal place area: recognition, navigation, or encoding? Neuron 23(1):115-125 (pii: S0896-6273(00)80758-8)

Epstein R, Deyoe EA, Press DZ, Rosen AC, Kanwisher N (2001) Neuropsychological evidence for a topographical learning mechanism in parahippocampal cortex. Cogn Neuropsychol 18(6):481-508. doi:10.1080/02643290125929

Epstein RA, Higgins JS, Thompson-Schill SL (2005) Learning places from views: variation in scene processing as a function of experience and navigational ability. $J$ Cogn Neurosci 17(1):73-83. doi:10.1162/0898929052879987

Fonteneau E, Bozic M, Marslen-Wilson WD (2015) Brain network connectivity during language comprehension: interacting linguistic and perceptual subsystems. Cereb Cortex 25(10):3962-3976. doi:10.1093/cercor/bhu283

Fox MD, Snyder AZ, Vincent JL, Corbetta M, Van Essen DC, Raichle ME (2005) The human brain is intrinsically organized into dynamic, anticorrelated functional networks. Proc Natl Acad Sci USA 102(27):9673-9678. doi:10.1073/pnas.0504136102

Fox MD, Corbetta M, Snyder AZ, Vincent JL, Raichle ME (2006) Spontaneous neuronal activity distinguishes human dorsal and ventral attention systems. Proc Natl Acad Sci USA 103(26):10046-10051. doi:10.1073/pnas.0604187103
Fox MD, Zhang D, Snyder AZ, Raichle ME (2009) The global signal and observed anticorrelated resting state brain networks. J Neurophysiol 101(6):3270-3283. doi:10.1152/jn.90777.2008

Freeman LC (1977) A set of measures of centrality based on betweenness. Am Sociol Assoc 40(1):35-41

Ghaem O, Mellet E, Crivello F, Tzourio N, Mazoyer B, Berthoz A, Denis M (1997) Mental navigation along memorized routes activates the hippocampus, precuneus, and insula. NeuroReport 8(3):739-744

Harvey CD, Coen P, Tank DW (2012) Choice-specific sequences in parietal cortex during a virtual-navigation decision task. Nature 484(7392):62-68. doi:10.1038/nature10918

He Y, Wang J, Wang L, Chen ZJ, Yan C, Yang H, Tang H, Zhu C, Gong Q, Zang Y, Evans AC (2009) Uncovering intrinsic modular organization of spontaneous brain activity in humans. PLoS One 4(4):e5226. doi:10.1371/journal.pone.0005226

Hegarty M, Richardson AE, Montello DR, Lovelace K, Subbiah I (2002) Development of a self-report measure of environmental spatial ability. Intelligence 30(5):425-447

Helfinstein SM, Schonberg T, Congdon E, Karlsgodt KH, Mumford JA, Sabb FW, Cannon TD, London ED, Bilder RM, Poldrack RA (2014) Predicting risky choices from brain activity patterns. Proc Natl Acad Sci USA 111(7):2470-2475. doi:10.1073/pnas.1321728111

Humphries MD, Gurney K (2008) Network 'small-world-ness': a quantitative method for determining canonical network equivalence. PLoS One 3(4):e0002051. doi:10.1371/journal.pone. 0002051

Igloi K, Doeller CF, Berthoz A, Rondi-Reig L, Burgess N (2010) Lateralized human hippocampal activity predicts navigation based on sequence or place memory. Proc Natl Acad Sci USA 107(32):14466-14471. doi:10.1073/pnas.1004243107

Ikkai A, Curtis CE (2011) Common neural mechanisms supporting spatial working memory, attention and motor intention. Neuropsychologia 49(6):1428-1434. doi:10.1016/j.neuropsycholo gia.2010.12.020

Jahn K, Deutschlander A, Stephan T, Strupp M, Wiesmann M, Brandt $\mathrm{T}$ (2004) Brain activation patterns during imagined stance and locomotion in functional magnetic resonance imaging. Neuroimage 22(4):1722-1731. doi:10.1016/j.neuroimage.2004.05. 017

Janzen G, Jansen C, van Turennout M (2008) Memory consolidation of landmarks in good navigators. Hippocampus 18(1):40-47. doi:10.1002/hipo.20364

Jenkinson M, Smith S (2001) A global optimisation method for robust affine registration of brain images. Med Image Anal 5(2):143-156 (pii: S1361841501000366)

Jenkinson M, Bannister P, Brady M, Smith S (2002) Improved optimization for the robust and accurate linear registration and motion correction of brain images. Neuroimage 17(2):825-841 (pii: S1053811902911328)

Kim JG, Aminoff EM, Kastner S, Behrmann M (2015) A neural basis for developmental topographic disorientation. J Neurosci 35(37):12954-12969

Kong XZ, Zhen Z, Li X, Lu HH, Wang R, Liu L, He Y, Zang Y, Liu J (2014) Individual differences in impulsivity predict head motion during magnetic resonance imaging. PLoS One 9(8):e104989. doi:10.1371/journal.pone.0104989

Kong XZ, Huang Y, Zhen Z, Huang L, Wang X, Yang Z, Liu J (2016a) Sex-linked association between cortical scene selectivity and spatial navigation ability (submitted)

Kong XZ, Song Y, Zhen Z, Liu J (2016b) Genetic variation in S100B modulates neural processing of visual scenes in Han Chinese. Cereb Cortex. doi:10.1093/cercor/bhv322

Kozlowski LT, Bryant KJ (1977) Sense-of-direction, spatial orientation, and cognitive maps. J Exp Psychol Hum Percept Perform 3(4):590-598 
Lebedev AV, Westman E, Simmons A, Lebedeva A, Siepel FJ, Pereira JB, Aarsland D (2014) Large-scale resting state network correlates of cognitive impairment in Parkinson's disease and related dopaminergic deficits. Front Syst Neurosci 8:45. doi:10. 3389/fnsys.2014.00045

Li Y, Liu Y, Li J, Qin W, Li K, Yu C, Jiang T (2009) Brain anatomical network and intelligence. PLoS Comput Biol 5(5):e1000395. doi:10.1371/journal.pcbi.1000395

Maguire EA (2001) The retrosplenial contribution to human navigation: a review of lesion and neuroimaging findings. Scand $\mathbf{J}$ Psychol 42(3):225-238

Maguire EA, Burgess N, Donnett JG, Frackowiak RS, Frith CD, O'Keefe J (1998) Knowing where and getting there: a human navigation network. Science 280(5365):921-924

Maguire EA, Gadian DG, Johnsrude IS, Good CD, Ashburner J, Frackowiak RS, Frith CD (2000) Navigation-related structural change in the hippocampi of taxi drivers. Proc Natl Acad Sci USA 97(8):4398-4403. doi:10.1073/pnas.070039597

Marchette SA, Vass LK, Ryan J, Epstein RA (2014) Anchoring the neural compass: coding of local spatial reference frames in human medial parietal lobe. Nat Neurosci 17(11):1598-1606. doi: $10.1038 / \mathrm{nn} .3834$

Maslov S, Sneppen K (2002) Specificity and stability in topology of protein networks. Science 296(5569):910-913. doi:10.1126/ science. 1065103

Mellet E, Tzourio N, Denis M, Mazoyer B (1995) A positron emission tomography study of visual and mental spatial exploration. J Cogn Neurosci 7(4):433-445. doi:10.1162/jocn.1995.7.4.433

Mendez MF, Cherrier MM (2003) Agnosia for scenes in topographagnosia. Neuropsychologia 41(10):1387-1395 (pii: S0028393203000411)

Mesulam MM, Mufson EJ (1982) Insula of the old world monkey. III: efferent cortical output and comments on function. J Comp Neurol 212(1):38-52. doi:10.1002/cne.902120104

Meunier D, Lambiotte R, Fornito A, Ersche KD, Bullmore ET (2009) Hierarchical modularity in human brain functional networks. Front Neuroinform 3:37. doi:10.3389/neuro.11.037.2009

Moffat SD, Kennedy KM, Rodrigue KM, Raz N (2007) Extrahippocampal contributions to age differences in human spatial navigation. Cereb Cortex 17(6):1274-1282. doi:10.1093/cercor/ bhl036

Mullally SL, Maguire EA (2011) A new role for the parahippocampal cortex in representing space. J Neurosci 31(20):7441-7449. doi:10.1523/JNEUROSCI.0267-11.2011

Murphy K, Birn RM, Handwerker DA, Jones TB, Bandettini PA (2009) The impact of global signal regression on resting state correlations: are anti-correlated networks introduced? Neuroimage 44(3):893-905. doi:10.1016/j.neuroimage.2008.09.036

Newman ME (2004) Fast algorithm for detecting community structure in networks. Phys Rev E Stat Nonlinear Soft Matter Phys 69(6 Pt 2):066133

Newman ME (2006) Modularity and community structure in networks. Proc Natl Acad Sci USA 103(23):8577-8582. doi:10.1073/pnas.0601602103

Newman ME, Girvan M (2004) Finding and evaluating community structure in networks. Phys Rev E Stat Nonlinear Soft Matter Phys 69(2 Pt 2):026113

O'Craven KM, Kanwisher N (2000) Mental imagery of faces and places activates corresponding stimulus-specific brain regions. J Cogn Neurosci 12(6):1013-1023

Ohnishi T, Matsuda H, Hirakata M, Ugawa Y (2006) Navigation ability dependent neural activation in the human brain: an fMRI study. Neurosci Res 55(4):361-369. doi:10.1016/j.neures.2006.04.009

O'Keefe J, Dostrovsky J (1971) The hippocampus as a spatial map. Preliminary evidence from unit activity in the freely-moving rat. Brain Res 34(1):171-175. doi:10.1016/0006-8993(71)90358-1
Onoda K, Yamaguchi S (2013) Small-worldness and modularity of the resting-state functional brain network decrease with aging. Neurosci Lett 556:104-108. doi:10.1016/j.neulet.2013.10.023

Park S, Chun MM (2009) Different roles of the parahippocampal place area (PPA) and retrosplenial cortex (RSC) in panoramic scene perception. Neuroimage 47(4):1747-1756. doi:10.1016/j. neuroimage.2009.04.058

Poldrack RA (2011) Inferring mental states from neuroimaging data: from reverse inference to large-scale decoding. Neuron 72(5):692-697. doi:10.1016/j.neuron.2011.11.001

Power JD, Barnes KA, Snyder AZ, Schlaggar BL, Petersen SE (2012) Spurious but systematic correlations in functional connectivity MRI networks arise from subject motion. Neuroimage 59(3):2142-2154. doi:10.1016/j.neuroimage.2011.10.018

Raven J (ed) (1995) Advanced progressive matrices sets I and II. Oxford Psychologist Press Ltd, Oxford

Rubinov M, Sporns O (2010) Complex network measures of brain connectivity: uses and interpretations. Neuroimage 52(3):1059-1069. doi:10.1016/j.neuroimage.2009.10.003

Sala-Llonch R, Junque C, Arenaza-Urquijo EM, Vidal-Pineiro D, Valls-Pedret C, Palacios EM, Domenech S, Salva A, Bargallo N, Bartres-Faz D (2014) Changes in whole-brain functional networks and memory performance in aging. Neurobiol Aging 35(10):2193-2202. doi:10.1016/j.neurobiolaging.2014.04.007

Schedlbauer AM, Copara MS, Watrous AJ, Ekstrom AD (2014) Multiple interacting brain areas underlie successful spatiotemporal memory retrieval in humans. Sci Rep 4:6431. doi:10.1038/ srep06431

Shepard RN, Metzler J (1971) Mental rotation of three-dimensional objects. Science 171(3972):701-703

Sholl MJ (1988) The relationship between sense of direction and mental geographic updating. Intelligence 12(3):299-314

Simon HA (1962) The architecture of complexity. Proc Am Philos Soc 106(6):467-482

Sobel DF, Gallen CC, Schwartz BJ, Waltz TA, Copeland B, Yamada S, Hirschkoff EC, Bloom FE (1993) Locating the central sulcus: comparison of MR anatomic and magnetoencephalographic functional methods. AJNR Am J Neuroradiol 14(4):915-925

Spiers HJ, Maguire EA (2006) Thoughts, behaviour, and brain dynamics during navigation in the real world. Neuroimage 31(4):1826-1840. doi:10.1016/j.neuroimage.2006.01.037

Sporns O, Tononi G, Kotter R (2005) The human connectome: a structural description of the human brain. PLoS Comput Biol 1(4):e42. doi:10.1371/journal.pcbi.0010042

Stam CJ, Reijneveld JC (2007) Graph theoretical analysis of complex networks in the brain. Nonlinear Biomed Phys 1(1):3. doi:10. 1186/1753-4631-1-3

Stevens AA, Tappon SC, Garg A, Fair DA (2012) Functional brain network modularity captures inter- and intra-individual variation in working memory capacity. PLoS One 7(1):e30468. doi:10. 1371/journal.pone. 0030468

Takahashi N, Kawamura M, Shiota J, Kasahata N, Hirayama K (1997) Pure topographic disorientation due to right retrosplenial lesion. Neurology 49(2):464-469

Tyler LK, Marslen-Wilson W (2008) Fronto-temporal brain systems supporting spoken language comprehension. Philos Trans R Soc Lond B Biol Sci 363(1493):1037-1054. doi:10.1098/rstb.2007. 2158

Tzourio-Mazoyer N, Landeau B, Papathanassiou D, Crivello F, Etard O, Delcroix N, Mazoyer B, Joliot M (2002) Automated anatomical labeling of activations in SPM using a macroscopic anatomical parcellation of the MNI MRI single-subject brain. Neuroimage 15(1):273-289. doi:10.1006/nimg.2001.0978

Valenstein E, Bowers D, Verfaellie M, Heilman KM, Day A, Watson RT (1987) Retrosplenial amnesia. Brain 110(Pt 6):1631-1646 
van den Heuvel MP, Sporns O (2011) Rich-club organization of the human connectome. J Neurosci 31(44):15775-15786. doi:10. 1523/JNEUROSCI.3539-11.2011

van den Heuvel MP, Sporns O (2013) Network hubs in the human brain. Trends Cogn Sci 17(12):683-696. doi:10.1016/j.tics.2013. 09.012

van den Heuvel MP, Stam CJ, Kahn RS, Hulshoff Pol HE (2009) Efficiency of functional brain networks and intellectual performance. J Neurosci 29(23):7619-7624. doi:10.1523/JNEUR OSCI.1443-09.2009

Van Dijk KR, Sabuncu MR, Buckner RL (2012) The influence of head motion on intrinsic functional connectivity MRI. Neuroimage 59(1):431-438. doi:10.1016/j.neuroimage.2011.07.044

Vann SD, Aggleton JP, Maguire EA (2009) What does the retrosplenial cortex do? Nat Rev Neurosci 10(11):792-802. doi:10.1038/nrn2733

Wang J, Wang X, Xia M, Liao X, Evans A, He Y (2015) GRETNA: a graph theoretical network analysis toolbox for imaging connectomics. Front Hum Neurosci 9:386. doi:10.3389/fnhum.2015. 00386

Watts DJ, Strogatz SH (1998) Collective dynamics of 'small-world' networks. Nature 393(6684):440-442. doi:10.1038/30918

Watrous AJ, Tandon N, Conner CR, Pieters T, Ekstrom AD (2013) Frequency-specific network connectivity increases underlie accurate spatiotemporal memory retrieval. Nat Neurosci 16(3):349-356. doi:10.1038/nn.3315

Wegman J, Janzen G (2011) Neural encoding of objects relevant for navigation and resting state correlations with navigational ability. J Cogn Neurosci 23(12):3841-3854. doi:10.1162/jocn_ a_00081

Wegman J, Fonteijn HM, van Ekert J, Tyborowska A, Jansen C, Janzen G (2014) Gray and white matter correlates of navigational ability in humans. Hum Brain Mapp 35(6):2561-2572. doi: 10.1002/hbm.22349

Wolbers T, Hegarty M (2010) What determines our navigational abilities? Trends Cogn Sci 14(3):138-146. doi:10.1016/j.tics. 2010.01.001

Xia M, Wang J, He Y (2013) BrainNet Viewer: a network visualization tool for human brain connectomics. PLoS One 8(7):e68910. doi:10.1371/journal.pone.0068910

Yarkoni T, Poldrack RA, Nichols TE, Van Essen DC, Wager TD (2011) Large-scale automated synthesis of human functional neuroimaging data. Nat Methods 8(8):665-670. doi:10.1038/ nmeth. 1635

Zeng LL, Wang D, Fox MD, Sabuncu M, Hu D, Ge M, Buckner RL, Liu H (2014) Neurobiological basis of head motion in brain imaging. Proc Natl Acad Sci USA. doi:10.1073/pnas. 1317424111

Zhen Z, Yang Z, Huang L, Kong XZ, Wang X, Dang X, Huang Y, Song Y, Liu J (2015) Quantifying interindividual variability and asymmetry of face-selective regions: a probabilistic functional atlas. Neuroimage 113:13-25. doi:10.1016/j.neuroimage.2015. 03.010 